\title{
Expanded polytetrafluoroethylene right ventricle to pulmonary artery conduit: Time to adopt?
}

\author{
Ming-Sing Si, MD
}

\footnotetext{
From the Section of Pediatric Cardiovascular Surgery, Department of Cardiac Surgery, University of Michigan, Ann Arbor, Mich.

Author has nothing to disclose with regard to commercial support.

Received for publication May 7, 2018; revisions received May 7, 2018; accepted for publication May 8, 2018; available ahead of print June 14, 2018

Address for reprints: Ming-Sing Si, MD, Section of Pediatric Cardiovascular Surgery, Department of Cardiac Surgery, 11-735 C.S. Mott Children's Hospital SPC 4204, 1540 E Hospital Dr, Ann Arbor, MI 48109-4204 (E-mail: mingsing@umich.edu).

J Thorac Cardiovasc Surg 2018;156:1637-8

$0022-5223 / \$ 36.00$

Copyright (c) 2018 by The American Association for Thoracic Surgery

https://doi.org/10.1016/j.jtcvs.2018.05.020
}

Establishment of right ventricle to pulmonary artery (RVPA) continuity is required in the surgical repair of many congenital heart defects. RVPA conduits have 2 main functions: provide an unobstructed connection between RV and PA, and prevent retrograde flow into the RV during diastole. Current options for RVPA conduits include pulmonary allograft, aortic allograft, xenograft valves in synthetic grafts or xenograft vessels, and custom-made expanded polytetrafluoroethylene (ePTFE) valves in ePTFE conduits.

RVPA conduits, because of their inability to grow and regenerate, will require replacement because of degeneration, calcification, and lack of somatic growth. Other problems of RVPA conduits are that they can incite an immunogenic response (allo- or xenograft) and are susceptible to infection. Although somatic growth is something that we can never expect from a synthetic, nondegradeable conduit, minimizing degeneration and calcification can be something that can be addressed with material selection.

Because of a lack of allograft material, surgeons in Asia have resorted to innovative approaches to create synthetic conduits. Miyazaki and colleagues across Japan $^{1}$ have accrued a large experience with ePTFE RVPA conduits. ${ }^{1}$ Yamamoto and colleagues ${ }^{2}$ present the midterm results of the second iteration of this ePTFE RVPA conduit. This conduit is created from thin ePTFE that is more compliant and thus may improve leaflet mobility, increase valve opening area, and decrease transvalve gradients. These potential improvements may be especially important for the smaller conduits needed in small patients.

In the study by Yamamoto and colleagues ${ }^{2}$, the investigators present their experience with their first-generation (normal thickness $[\mathrm{n}=55]$ ) and second-generation (decreased thickness $[\mathrm{n}=94]$ ) ePTFE RVPA conduits in 149 patients with a median follow up of 2.9 years. Subgroup analysis was performed in conduits of different sizes, and the ePTFE RVPA conduits were implanted in children with a variety of congenital heart defects. The

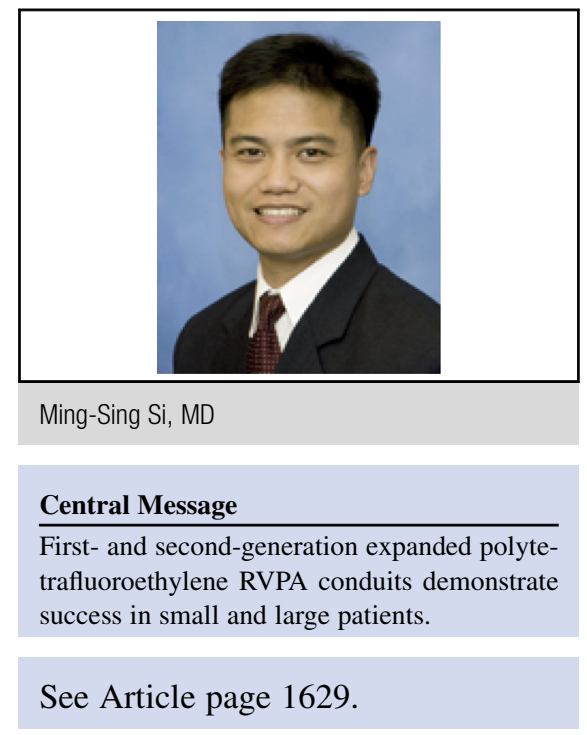

investigators found that the ePTFE RVPA conduits in general performed quite well with a low incidence of regurgitation beyond moderate on echocardiogram. Conduits tended to become stenotic, likely due to lack of somatic growth. When considering the entire cohort, there was no difference between the normal and thin conduits in terms of regurgitation or gradient. However, subgroup analysis revealed that for the smaller conduits, those of normal thickness had higher gradients and for the larger conduits, the thin ones had more regurgitation. The authors therefore conclude that the secondgeneration RVPA conduits made from thin ePTFE are more appropriate for smaller patients.

Should surgeons in other parts of the world where allografts are available adopt this ePTFE RVPA conduit? Certainly, long-term results are needed for the large conduits because in these patients no somatic growth may be taking place and the durability of the valve will be a deciding factor in answering this question. Ootaki and colleagues ${ }^{3}$ recently presented their results in 26 patients with ePTFE RVPA conduits and demonstrated excellent short- to midterm results. Shinkawa and colleagues ${ }^{4}$ reported excellent short-term results with 160 patients. These results appear to replicate the experience of the Japanese groups that pioneered this innovation and thus the ePTFE RVPA conduit warrants further consideration for more widespread use and testing in a multicenter clinical trial that directly compares it with pulmonary allograft. 


\section{References}

1. Miyazaki T, Yamagishi M, Maeda Y, Yamamoto Y, Taniguchi S, Sasaki Y, et al. Expanded polytetrafluoroethylene conduits and patches with bulging sinuses and fan-shaped valves in right ventricular outflow tract reconstruction: multicenter study in Japan. J Thorac Cardiovasc Surg. 2011;142:1122-9.

2. Yamamoto Y, Yamagishi M, Miyazaki T, Asada S, Maeda Y, Yaku H, et al. Modification of expanded polytetrafluoroethylene valved conduit using the thin-type leaflets. J Thorac Cardiovasc Surg. 2018;156:1629-36.
3. Ootaki Y, Welch AS, Walsh MJ, Quartermain MD, Williams DA, Ungerleider RM. Medium-term outcomes after implantation of expanded polytetrafluoroethylene valved conduit. Ann Thorac Surg. 2018;105: 843-50.

4. Shinkawa T, Tang X, Gossett JM, Mustafa T, Hategekimana F, Watanabe F, et al. Valved polytetrafluoroethylene conduits for right ventricular outflow tract reconstruction. Ann Thorac Surg. 2015;100: 129-37. 